\title{
Naissance et métamorphoses du savoir démographique : le mestizaje des communautés indigènes de la Valle Bajo de Cochabamba, en Bolivie
} THE CREATION AND CHANGING DEFINITION OF DEMOGRAPHIC KNOWLEDGE: THE CASE OF MESTIZAJE IN THE CORPORATE INDIGENOUS COMMUNITIES OF THE VALLE BAJO OF COCHABAMBA, BOLIVIA

\author{
EL NACIMIENTO Y LAS METAMORFOSIS DEL SABER \\ DEMOGRAFICO, O EL MESTIZAJE DE LAS COMUNIDADES \\ INDIGENAS DEL VALLE BAJO DE COCHABAMBA, EN BOLIVIA
}

\author{
Robert H. Jackson
}

\section{Volume 25, numéro 1, printemps 1996}

Savoir démographique et pratique du pouvoir dans le Tiers Monde : perspectives historiques

URI : https://id.erudit.org/iderudit/010201ar

DOI : https://doi.org/10.7202/010201ar

Aller au sommaire du numéro

Éditeur(s)

Association des démographes du Québec

ISSN

0380-1721 (imprimé)

1705-1495 (numérique)

Découvrir la revue

Citer cet article

Jackson, R. H. (1996). Naissance et métamorphoses du savoir démographique : le mestizaje des communautés indigènes de la Valle Bajo de Cochabamba, en Bolivie. Cahiers québécois de démographie, 25(1), 69-99.

https://doi.org/10.7202/010201ar

\section{Résumé de l'article}

$\mathrm{Au}$ XVIe et au XVIIe siècle, les conquérants espagnols créèrent un éventail de dénominations raciales pour désigner et catégoriser, à des fins fiscales, les populations américaines d'ascendance mixte. Étant donné que ce système de castes reposait sur le postulat que la filiation sanguine était reconnaissable à la couleur de la peau et aux caractéristiques physiques, les renseignements démographiques recueillis sur ces bases Jurent constamment marqués par l'imprécision. De plus, leur sens se transforma au fil des décennies. Les prêtres et les agents de recensement interprétèrent les termes raciaux de façon subjective et les utilisèrent en fonction de leurs préférences individuelles. Par exemple, il arriva que dans les registres d'une paroisse, après l'arrivée ou le départ d'un prêtre, la fréquence de l'une ou l'autre catégorie raciale présente, à court terme, des variations significatives. Les changements socio-économiques modifièrent aussi, à la longue, le sens des termes raciaux et la définition des statuts qui y étaient rattachés. La Valle Ballo de Cochabamba, en Bolivie, illustre ce processus : la multiplication des mestizos dans les trois communautés indigènes étudiées ici ne fut pas strictement affaire de mélange racial et doit être mise en rapport avec l'évolution des identités et des statuts assignés aux indios.
Tous droits réservés @ Association des démographes du Québec, 1996
Ce document est protégé par la loi sur le droit d'auteur. L’utilisation des services d’Érudit (y compris la reproduction) est assujettie à sa politique d'utilisation que vous pouvez consulter en ligne.

https://apropos.erudit.org/fr/usagers/politique-dutilisation/ 
Cahiers québécois de démographie

Vol. 25, no 1, printemps 1996, p. 69-99.

\title{
Naissance et métamorphoses du savoir démogra- phique : le mestizaje des communautés indigènes de la Valle Bajo de Cochabamba, en Bolivie
}

\author{
Robert H. JACKSON *
}

Dès l'aube de la colonisation espagnole en Amérique, les fonctionnaires de la Couronne, les missionnaires et les agents de recensement instituèrent une hiérarchie de statuts légaux fondée sur la catégorisation des populations en fonction de la race. Les catégories raciales ainsi créées reposaient sur des définitions qui laissaient place à la connaissance subjective des caractéristiques biologiques et culturelles utilisées pour définir et classer les populations. Par ailleurs, la conjoncture sociale et économique et les impératifs de la politique royale firent évoluer ces définitions.

L'Amérique hispanique offre de nombreux exemples de politiques conçues pour créer de nouvelles identités et marquer les différences entre colonisateurs et colonisés. Ainsi, l'Espagne promulgua des lois somptuaires, réserva à la population indigène un statut et des droits fiscaux distincts de ceux des non indigènes, et instaura pour elle un système judiciaire parallèle. En outre, peu après la conquête, au XVIe siècle, les autorités coloniales établirent une ségrégation résidentielle stricte entre les deux populations. Dans la plupart des villes coloniales, on trouvait une traza, quartier résidentiel habituellement situé dans la zone entourant la grand-place de la ville et réservé aux blancs. Le gouvernement espagnol légiféra également pour empêcher les Espagnols d'origine et les castas (ou "castes" : aux termes de la loi, les personnes d'ascendance mixte, en qui

\footnotetext{
* Département d'histoire, de géographie et d'économique, Texas Southern University. Version française : Johanne Archambault, sous la supervision de Raymond R. Gervais.
} 
le sang européen se mélangeait au sang africain ou indigène) de vivre au sein des communautés indigènes. Enfin, il inscrivit dans la loi un système de castes qui fixait des distinctions entre la population marquée par une ascendance européenne prédominante et les populations de couleur, de souche indigène ou africaine, et exerça de multiples formes de discrimination contre les castas, leur fermant notamment l'accès à certains emplois et à de multiples corporations professionnelles.

Le gouvernement espagnol imposa également une capitation, appelée tribut, qui n'était censée frapper que les personnes classées comme indios. Indio devint ainsi un terme générique utilisé au premier chef pour déterminer le statut fiscal particulier des populations indigènes. L'éventail de catégories raciales défini par les Espagnols servit à étiqueter les populations d'ascendance mixte et à créer les distinctions légales et sociales sur lesquelles allait s'édifier la hiérarchie sociale - le système de castes - de la période coloniale. L'assignation de catégories raciales aux castas reposait sur le postulat que prêtres et fonctionnaires coloniaux pouvaient reconnaitre l'ascendance, ou plus précisément la filiation sanguine, à la couleur de la peau. Mais d'autres critères contribuèrent à la création des nouvelles identités raciales, en particulier certains stéréotypes relatifs à la culture et aux coutumes et, dans le cas des populations rurales, au mode d'occupation du sol. Les dénominations raciales rattachaient l'identité assignée à un statut légal précis.

Cette étude examine le phénomène de création d'identités et d'évolution de la définition du savoir démographique tel qu'il se dégage des registres paroissiaux de trois communautés indigènes de la Valle Bajo de Cochabamba, en Bolivie. Elle est centrée sur la dynamique du processus de mestizaje, c'est-à-dire de brassage racial souvent accompagné de transformations sociales et culturelles, à la faveur duquel prit naissance un groupe qui, au sein du système de castes espagnol, fut défini comme d'ascendance à la fois européenne et indigène, ou se vit assigner une position sociale et culturelle intermédiaire entre celle de l'Européen et celle de l'indigène. L'analyse du mestizaje fait ressortir l'imprécision du savoir démographique et montre comment une longue évolution sociale, économique et culturelle a modifié les définitions sur lesquelles reposaient les identités et les statuts déterminés par la loi.

Le système de castes colonial reposait sur le postulat qu'il existait des distinctions significatives entre individus de catégo- 
ries différentes ${ }^{1}$. Pourtant, la couleur de la peau n'impliquait pas à elle seule des différences de culture, dans la langue ou l'habillement par exemple, ni la persistance de coutumes comme l'ayni (échange de travail), pratiqué par les communautés paysannes andines. De plus, les identités n'avaient rien de figé, et les comportements individuels pouvaient rompre avec les stéréotypes qui fondaient la définition des catégories. Ce dernier aspect du processus de création d'identité se vérifie indéniablement dans le cas de la population indigène. Les indios pouvaient modifier leur tenue vestimentaire, apprendre l'espagnol, s'installer dans une ville ou quitter leur village d'origine, adopter une profession qui n'entrait pas dans leurs coutumes, et, reclassés comme mestizos, se voir exemptés des charges fiscales imposées à la population indigène. C'est ainsi qu'un fonctionnaire de la Couronne, Francisco de Viedma, intendant de Cochabamba, voulut compenser la perte de revenus fiscaux qui avait résulté, durant les années 1780 , de l'acquisition du statut de mestizo par des indios, en faisant valoir une nouvelle catégorie, celle de cholo, qui représentait en principe un stade intermédiaire entre les statuts d'indio et de mestizo. Le roi lui ayant ordonné d'augmenter les revenus produits par la colonie, il tenta de ranger les cholos dans les catégories assujetties au tribut.

Le progrès des idées et l'évolution des politiques des gouvernements à l'égard des populations indigènes au cours des XVIIle et XIXe siècles modifièrent également la définition des races et le processus de mestizaje en Bolivie. À l'époque coloniale, le gouvernement avait créé des villages indigènes assimilables à des réserves, qui permettaient de séparer les indios de la population non indigène, de leur fournir des terres dont les surplus de production pouvaient être prélevés en taxes et de les organiser en noyaux de population faciles à soumettre à la corvée. Durant les décennies qui suivirent l'indépendance, des

Certains chercheurs font écho aujourd'hui à ce genre de postulat à propos de peuples également classés dans des catégories raciales artificielles. Gootenberg (1991), analysant les flottements des statuts indio et mestizo chez les paysans du Pérou, n'échappe pas à la contradiction lorsqu'il écrit : "L'obscurité de la preuve incite à croire, dans l'ensemble, à la consolidation d'un nombre assez important de villages pseudo-indiens [...] où toutes sortes de paysans reproduisaient [c'est moi qui souligne] une bonne part des coutumes indigènes de mise en commun du travail et des ressources, par nécessité ou par intérêt". Il sous-entend qu'il existait des différences sociales et culturelles significatives entre les paysans que les agents de recensement inscrivaient soit comme indios soit comme mestizos. 
hommes politiques influencés par les philosophies libérales se firent les champions de la modernisation et de l'évolution sociale. La première moitié du XIXe siècle vit triompher les théories libérales qui prônaient la pleine intégration des indigènes à l'ensemble de la société; déjà, dans les pays andins, et ce jusqu'à la fin des années 1820, Simon Bolivar et ses lieutenants avaient fait la promotion d'un libéralisme à saveur utopique. On envisageait aussi de privatiser les terres communautaires des indigènes et de créer une classe de petits propriêtaires exploitants comme il en existait aux États-Unis, afin de jeter les bases d'un système politique républicain stable.

Plus tard au cours du siècle, le positivisme et surtout le darwinisme social, également venus d'Europe, gagnèrent de l'influence en Bolivie. Les indigènes faisaient désormais figure d'obstacle à la modernisation, et certains idéologues allèrent jusqu'à défendre l'idée de les exproprier pour confier leurs terres à une population non indigène mieux à même de les faire valoir $^{2}$. La mentalité qui prévalait alors officiellement à leur égard s'apparentait à celle qui, à peu près à la même époque, entraîna au Brésil la propagation du mythe du "blanchissement" de la population (diminution de sa composante afrobrésilienne) et l'adoption de mesures comme les quotas d'immigration pour accélérer le processus. Les recensements nationaux apparaissaient comme une sorte de message envoyé à l'Europe, et dans des pays comme la Bolivie, dont les fortes minorités indigènes pouvaient avoir l'air d'entacher le désir de modernisme et l'aspiration à un européanisme plus affirmé, il n'est pas impossible que les agents de recensement aient incliné à inscrire des indios comme mestizos pour diminuer, dans les recensements officiels où les mentors intellectuels des élites du pays lisaient la société bolivienne en raccourci, la visibilité d'une population indigène jugée arriérée. Effacer la population indigène n'était pas seulement affaire d'image de soi : c'était une opération susceptible d'influencer des investisseurs étrangers potentiels qui, imbus des théories raciales pseudoscientifiques de la fin du XIXe siècle, risquaient d'être moins portés à investir dans des pays ou des régions dont les fortes populations indigènes n'offraient pas une main-d'œuvre adéquate.

2 Voir par exemple Hale, 1968; Demelas, 1981; Langer, 1988; Langer et Jackson, 1990; Grieshaber, 1985. Pour une analyse de la pensée et des politiques sociales et raciales prévalant dans un autre pays latinoaméricain, voir Skidmore, 1974. 
Des années 1820 à 1870 , la classe politique bolivienne débattit de la privatisation et du fractionnement des terres communautaires, mais le projet ne commença à prendre forme qu'en 1874, avec l'adoption de la loi d'ex-vinculación, dont la mise en œuvre fut entreprise aux environs de l'année 1880 . Si l'intérêt du Trésor public pour le tribut et la résistance des collectivités indigènes au fractionnement de leurs terres en freinèrent l'application, il n'en demeure pas moins que ces efforts pour démanteler les institutions coloniales qui donnaient à l'identité indio ses assises raciales et légales précipitèrent le mestizaje. Du reste, les décennies immédiatement postérieures à l'indépendance de la Bolivie baignaient dans un climat nouveau, où il était plus facile de se dire mestizo là où l'on aurait été étiquetê indio au siècle précédent, surtout pour un paysan vivant hors des villages, sur une hacienda ou en ville. Les grands propriétaires fonciers, par exemple, s'arrangeaient pour faire retirer le nom de leurs ouvriers des listes de tributaires afin de ne pas avoir à payer le tribut pour eux ou à leur donner congé pour qu'ils aillent gagner ailleurs la somme nécessaire. La disparition graduelle du système du tribut dans la deuxième moitié du XIXe siècle et la mise en ouvre de la loi d'exvinculación provoquèrent l'élimination des listes de tributaires, ultimes dossiers officiels contenant le nom de ceux qui avaient le statut d'indio astreint au tribut. Et à mesure que le siècle avançait vers sa fin et que la société rurale et l'économie de Cochabamba se transformaient, les éléments de l'identité indio changeaient aussi.

La Valle Bajo de Cochabamba est un cas intêressant pour comprendre la nature et le cours du mestizaje. Sa population rurale était principalement composée de personnes définies comme indios ou comme mestizos. Au point de vue du statut social, les mestizos se situaient entre les españoles, pour la plupart blancs et le plus souvent membres de la strate supérieure, et les indios. Le statut indio était assez clairement défini durant la plus grande partie de la période coloniale, reposant sur le costume, la langue, la profession et surtout le lieu de résidence, soit l'une des quatre communautés indigènes de la vallée. Mais il n'était pas immuable : pour y échapper, il suffisait de modifier les éléments entrant dans la définition. Ainsi, en quittant son village d'origine, un indio pouvait se libérer à la fois de son statut et des obligations qui s'y rattachaient.

Au XVIIIe et au XIXe siècle, après l'indépendance de la Bolivie (1825), le mestizaje s'accéléra dans la Valle Bajo au point 
que, vers 1900 , les fonctionnaires chargés de confectionner le premier véritable recensement national de l'histoire du pays inscrivirent comme mestizos la majorité des habitants de trois des plus grands districts de la vallee, y compris les communautés indigènes qui nous intéressent. Les registres d'état civil de ces dernières attestent le processus de mestizaje.

On constate en effet, dans les registres des baptêmes, une évolution de la distribution des nouveau-nés inscrits comme indios et comme mestizos. Les archives paroissiales les plus complètes qui aient été conservées sont celles de Passo. À la fin des années 1760, les baptêmes de nouveau-nés inscrits comme indios y comptent pour 76,6 pour cent de l'ensemble et les baptêmes de mestizos pour seulement 17,2 pour cent. Soixante ans plus tard, à la fin des années 1820 , la fréquence des baptêmes de nouveau-nés indios n'est plus que de 48,5 pour cent, comparativement à 42,8 pour cent de mestizos. Encore 80 ans plus tard, durant la première décennie du XXe siècle, 89,9 pour cent des nouveau-nés sont inscrits comme mestizos au moment de leur baptême et seulement 2,8 pour cent apparaissent comme indios (voir le tableau 1).

TABLEAU 1 - Distribution des baptêmes au sein de la population indigène et non indigène de la paroisse de Passo, par catégorie raciale (en pourcentage)

\begin{tabular}{lrrrrrr}
\hline & $1747-$ & $1767-$ & $1824-$ & $1834-$ & $1859-$ & $1869-$ \\
Catégorie & 1751 & 1771 & 1828 & 1838 & 1863 & 1873 \\
\hline Indigènes & & & & & & \\
(tous) & 75,8 & 76,6 & 48,5 & 40,3 & 42,5 & 33,3 \\
Español & 4,8 & 3,5 & 7,3 & 3,8 & 8,6 & 6,9 \\
Mestizo & 13,5 & 17,2 & 42,8 & 54,9 & 48,1 & 59,6 \\
Cholo & - & 2,1 & - & - & - & - \\
Mulato & - & 0,7 & 0,3 & - & - & - \\
Information & & & & & & 0,1 \\
manquante & 5,9 & - & 1,2 & 0,9 & 0,8 & 0,1 \\
\hline Catégorie & $1903-$ & $1912-$ & & & & \\
& 1907 & 1916 & & & & \\
\hline Indio & 2,5 & 6,8 & & & & \\
Español & 5,2 & - & & & & \\
Blanco & 2,5 & 6,3 & & & & \\
Mestizo & 89,9 & 86,9 & & & & \\
\hline
\end{tabular}

Source : APP. 
TABLEAU 2 - Distribution des baptêmes au sein de la population indigène et non indigène de la paroisse de SipeSipe, par catégorie raciale (en pourcentage)

\begin{tabular}{lrrrrrr}
\hline Catégorie & $1745-$ & $1755-$ & $1765-$ & $1774-$ & $1784-$ & $1815-$ \\
\hline Indiens & 1749 & 1759 & 1769 & 1778 & 1788 & 1819 \\
(tous) & 70,2 & 63,0 & 60,7 & 59,4 & 47,8 & 43,7 \\
Español & 6,2 & 6,1 & 9,0 & 8,5 & 9,0 & 6,2 \\
Mestizo & 19,9 & 28,7 & 29,5 & 31,6 & 40,0 & 48,9 \\
Mulato & 0,2 & 0,1 & 0,5 & 0,3 & 0,9 & 0,3 \\
Sambo & 0,2 & - & - & - & 0,2 & - \\
Esclave & 0,2 & - & - & - & - & - \\
Moso & - & - & - & - & 1,9 & - \\
Information & & & & & & \\
manquante & 3,3 & 2,1 & 0,4 & 0,2 & 0,1 & 0,9 \\
\hline
\end{tabular}

Source : APS.

Les registres des baptêmes des trois autres communautés de la Valle Bajo témoignent de la même évolution, bien qu'ils ne soient pas aussi complets. À SipeSipe, la part des baptêmes d'enfants définis comme indios, de 70,2 pour cent à la fin des années 1740 , passe à 43,7 pour cent à la fin des années 1820 . Simultanément, la proportion passe de 19,9 à 48,9 pour cent pour les mestizos (voir le tableau 2). Dans le cas de Tiquipaya, les registres laissent voir, entre la fin des années 1740 et les années 1830 , une chute des baptêmes de 76,5 à 34,2 pour cent dans le cas des indios et une augmentation de 11,5 à 58,6 pour cent dans le cas des mestizos (tableau 3). Le dernier exemple, tiré des registres de Colcapirhua, va dans le même sens : atteignant 62,9 pour cent au début des années 1780 , les baptêmes d'indios se situent à 39,3 pour cent dans les années 1820 , tandis que les baptêmes de mestizos passent de 23,8 à 46,9 pour cent (voir le tableau 4).

Au fil du temps, la même transition du statut d'indio à celui de mestizo est mise en évidence par des recensements divers, tels le dénombrement effectué par l'intendant Francisco de Viedma à la fin des années 1780 et le recensement national de 1900 , qui comptent parmi les recensements généraux, et les recensements paroissiaux de SipeSipe (1798) et de Passo (1823). De l'ensemble ressort une tendance au déclin numérique des indios relativement aux mestizos (tableau 5). En ce qui concerne la Valle Bajo, on constate que la population indi- 
TABLEAU 3 - Distribution des baptêmes au sein de la population indigène et non indigène de la paroisse de Tiquipaya, par catégorie raciale (en pourcentage)

\begin{tabular}{lcccc}
\hline & $1745-$ & $1758-$ & $1768-$ & $1830-$ \\
Catégorie & 1749 & 1762 & 1772 & 1838 \\
\hline Indio & 76,5 & 75,8 & 70,3 & 34,2 \\
Originario & 1,5 & - & - & - \\
Español & 9,6 & 5,9 & 5,6 & 7,2 \\
Mestizo & 11,5 & 16,3 & 23,1 & 58,6 \\
Mulato & 0,3 & 0,5 & - & -05 \\
Pardo & - & - & 0,2 & - \\
Information & & 1,4 & 0,9 & - \\
manquante & 0,4 & & & \\
\hline
\end{tabular}

Source : APT.

TABLEAU 4 - Distribution des baptêmes au sein de la population indigène et non indigène de la vice-paroisse de Colcapirhua, par catégorie raciale (en pourcentage)

\begin{tabular}{lcrrrr}
\hline & $1779-$ & $1792-$ & $1803-$ & $1813-$ & $1823-$ \\
Catëgorie & 1783 & 1796 & 1807 & 1817 & 1827 \\
\hline Indigènes (tous) & 62,9 & 59,5 & 52,0 & 49,3 & 39,3 \\
Español & 12,9 & 7,5 & 9,9 & 13,3 & 13,7 \\
Mestizo & 23,8 & 32,9 & 37,3 & 37,2 & 46,9 \\
Mulato & - & - & - & 0,2 & 0,2 \\
Information & & & & - & - \\
manquante & 0,5 & - & 0,8 & - & \\
\hline
\end{tabular}

Source : APC.

TABLEAU 5 - Distribution de la population de trois communautés de la Valle Bajo, par catégorie raciale (en pourcentage)

\begin{tabular}{|c|c|c|c|c|c|c|c|c|}
\hline \multirow[b]{2}{*}{ Catégorie } & \multicolumn{3}{|c|}{ Passo } & \multicolumn{3}{|c|}{ SipeSipe } & \multicolumn{2}{|c|}{ Tiquipaya } \\
\hline & 1788 & 1823 & 1900 & 1788 & 1798 & 1900 & 1788 & 1900 \\
\hline Indio & 64,6 & 55,1 & 34,0 & 55,7 & 41,3 & 28,4 & 39,9 & 18,0 \\
\hline Español/Blanco & 8,1 & 9,1 & 6,0 & 11,6 & 3,3 & 12,7 & 11,8 & 20,2 \\
\hline Mestizo & 15,5 & 35,3 & 53,4 & 21,9 & 35,2 & 50,3 & 20,5 & 55,1 \\
\hline Cholo & 4,1 & - & - & 6,0 & - & - & 21,2 & - \\
\hline Mulato & 7,8 & - & 0,1 & 4,8 & 1,4 & - & 6,6 & - \\
\hline Information & & & & & & & & \\
\hline manquante & - & 0,5 & 6,5 & - & 18,7 & 8,5 & - & 6,7 \\
\hline
\end{tabular}

Source : Viedma, 1969 : 67; S. n.; Jackson et Maddox, 1993; Gordillo Claure, 1987. 
TABLEAU 6 - Population indigène de quatre communautés de la Valle Bajo, diverses années

\begin{tabular}{lccrc}
\hline Année & Passo & SipeSipe & Tiquipaya & Colcapirhua \\
\hline 1573 & 3298 & 3591 & 2657 & - \\
1683 & 1350 & 1573 & 778 & - \\
1788 & 1230 & 2017 & 1735 & $*$ \\
1798 & - & 2375 & - & - \\
1823 & 1311 & - & - & - \\
1900 & 1516 & 2648 & 819 & 539 \\
\hline
\end{tabular}

Source: Sanchez-Albornoz, 1978 : 29; Viedma, $1969: 67$; S. n.; Oficina Nacional de Inmigracion, Estadistica, y Propaganda Geografica, 1902.

* Information manquante.

gène enregistre une légère croissance absolue à Passo et à SipeSipe entre 1780 et 1900 , mais subit un déclin significatif à Tiquipaya (tableau 6). Une lecture superficielle des chiffres pourrait faire conclure à la stagnation de la population indigène au cours de la période et à une progression modérée et même élevée de la population mestizo.

Tel qu'il ressort des facteurs susceptibles de l'expliquer, le rapide mestizaje de la Valle Bajo est à la fois une réalitê socioculturelle et un tour de prestidigitation. Le déclin relatif de la population indigène sur le papier va de pair, en effet, avec l'évolution de la définition de l'indio et du mestizo. Nous avons souligné l'imprécision du statut d'indio. Les prêtres qui baptisaient savaient très bien ce qu'était un indio, mais ne se sont jamais entendus sur la terminologie à employer pour décrire les peuples indigènes. Les termes qui leur servaient à définir le statut des non indigènes ont évolué tout aussi bien, et là encore chacun les choisissait à sa manière.

\section{LES FLUCTUATIONS DE L'USAGE}

En vertu du statut fiscal spécifique dont l'avaient dotée les Espagnols, la population indigène des Amériques était couramment désignée par le générique indio, qui confondait des groupes divers au plan ethnique et linguistique dans une même entité définie par l'obligation de payer le tributo et astreinte, dans certains districts andins, à la corvée aux mines (mita). La fréquence des migrations dans les Andes au XVIe et au XVIIe siècle fit apparaître de nouveaux groupes de tributaires, classés 
par le fisc comme forasteros (personnes absentes de leur communauté d'origine) ou comme agregados (personnes absentes de leur communauté d'origine et établies dans une autre communauté); ces migrants, dont l'accès au sol était moins assuré, se voyaient imposer un tribut moins lourd. Les membres des communautés, qui jouissaient du plein accès aux terres communautaires et payaient un tribut plus élevé, étaient appelés originarios. Enfin, les yanaconas, dépourvus de lien avec une communauté, exécutaient toutes sortes de travaux pour les Espagnols, généralement à titre d'esclaves.

La terminologie fiscale servant à déterminer le statut des indigènes au regard du tribut était parfois employée, au moment de l'enregistrement des baptêmes, par les prêtres en poste dans les communautés de la Valle Bajo pour définir le statut des nouveau-nés selon celui de leurs parents, mais tous ne suivaient pas cette pratique. C'est pourquoi la disparition des catégories fiscales dans les registres des baptêmes ne signifiait pas la fin du classement des indigènes en fonction de leur statut fiscal, mais plutôt l'abandon de ces dénominations par les prêtres, souvent en faveur du générique indio.

À la longue, il y eut aussi, de l'une à l'autre et à l'intérieur des quatre communautés qui nous intéressent, beaucoup de variations dans les termes appliqués à la population non indigène. Au XVIIIe siècle, les prêtres de Passo, par exemple, en utilisèrent plusieurs, dont les plus courants étaient español, pour les personnes nées en Espagne et pour les blancs nés en Amérique, et mestizo, habituel pour les descendants d'unions entre conjoints d'ascendance européenne et d'ascendance indigène. Enfin, une population peu nombreuse, de souche africaine, répondait aux termes pardo, mulato, sambo, negro et esclavo (voir le tableau 7).

Quelques termes, affectionnés par certains prêtres durant plusieurs décennies, disparurent ensuite des registres paroissiaux, tel cholo, nom désignant à l'époque un descendant moins acculturé d'un mestizo et d'un indio et d'usage courant aujourd'hui pour la population bolivienne d'ascendance mixte. Cholo n'apparaît que peu de temps dans les registres paroissiaux des baptêmes et des mariages de Passo, et sa petite histoire illustre bien l'impact de la politique sur l'évolution et l'usage des termes raciaux et sur l'identité raciale. Dans son fameux rapport de 1788, l'intendant Francisco de Viedma n'enregistre des cholos que dans deux districts de Cochabamba : celui de Tapacari, où se situait la Valle Bajo, et celui d'Arque, l'un et l'autre 
TABLEAU 7 - Terminologie utilisée pour désigner la population non indigène de la paroisse de Passo

\begin{tabular}{|c|c|c|c|c|c|c|c|}
\hline $\begin{array}{l}1727- \\
1731\end{array}$ & $\begin{array}{l}1737- \\
1741\end{array}$ & $\begin{array}{l}1747- \\
1751\end{array}$ & $\begin{array}{l}1757- \\
1761\end{array}$ & $\begin{array}{l}1767- \\
1771\end{array}$ & $\begin{array}{l}1824- \\
1828\end{array}$ & $\begin{array}{l}1834- \\
1838\end{array}$ & $\begin{array}{l}1859- \\
1863\end{array}$ \\
\hline $\begin{array}{l}\text { Español } \\
\text { Mestizo } \\
\text { Pardo } \\
\text { Esclavo }\end{array}$ & $\begin{array}{l}\text { Español } \\
\text { Mestizo }\end{array}$ & $\begin{array}{l}\text { Español } \\
\text { Mestizo } \\
\text { Sambo } \\
\text { Mulato }\end{array}$ & $\begin{array}{l}\text { Español } \\
\text { Mestizo } \\
\text { Cholo } \\
\text { Mulato } \\
\text { Negro }\end{array}$ & $\begin{array}{l}\text { Español } \\
\text { Mestizo } \\
\text { Cholo } \\
\text { Mulato }\end{array}$ & $\begin{array}{l}\text { Español } \\
\text { Mestizo } \\
\text { Mulato }\end{array}$ & $\begin{array}{l}\text { Español } \\
\text { Blanco } \\
\text { Mestizo }\end{array}$ & $\begin{array}{l}\text { Español } \\
\text { Blanco } \\
\text { Mestizo }\end{array}$ \\
\hline $\begin{array}{l}1869- \\
1873\end{array}$ & $\begin{array}{l}1903- \\
1907\end{array}$ & $\begin{array}{l}1912- \\
1916\end{array}$ & & & & & \\
\hline $\begin{array}{l}\text { Español } \\
\text { Blanco } \\
\text { Mestizo }\end{array}$ & $\begin{array}{l}\text { Blanco } \\
\text { Español } \\
\text { Mestizo }\end{array}$ & $\begin{array}{l}\text { Blanco } \\
\text { Español } \\
\text { Mestizo }\end{array}$ & & & & & \\
\hline
\end{tabular}

Source : APP.

caractérisés par la présence de communautés indigènes et de fortes populations d'originarios et de forasteros. L'utilisation de cholo par Viedma témoigne de son pragmatisme fiscal. Les cholos étant soumis au tribut, l'intendant espérait manifestement ramener les mestizos au statut de tributaires en les assimilant à cette catégorie (Larson, 1988: 11). Mais cholo apparait rarement dans d'autres registres.

Il figure pour la première fois dans celui des baptêmes de Passo ${ }^{3}$, en 1758, et l'échantillon de 1757-1761 compte au total 138 baptêmes d'enfants inscrits comme cholos. L'échantillon suivant (1767-1771) n'en contient que treize, puis le terme disparaît. Aucun prêtre en poste à SipeSipe et à Colcapirhua ne l'utilisa jamais ${ }^{4}$. Il faudrait pousser davantage les recherches pour comprendre les origines de son usage actuel à Cochabamba, mais l'opinion que les cholos formaient une part importante de la population de Cochabamba paraît remonter à un

3 Registres paroissiaux des baptèmes de Passo, tirés des Archives paroissiales de Passo, Passo, Bolivie (APP dans la suite du texte); nous citons de la même manière les Registres paroissiaux des baptêmes de Colcapirhua (APC), de SipeSipe (APS) et de Tiquipaya (APT). La référence complète de chacun des sigles figure dans les références bibliographiques. Cholo apparaît aussi une fois dans les échantillons tirés de Tiquipaya pour 1747. Le prêtre a utilisé le terme cholo dans la marge du registre où il a inscrit le nom de l'enfant baptisé, mais le terme mestizo dans l'acte de baptême lui-même. 
document de l'ère coloniale, de nouveau le rapport de Viedma, qui enregistre une population de 1449 cholos dans la Valle Bajo au cours des années 1780, dont 78 à Passo, 218 à SipeSipe et 922 à Tiquipaya (1969: 66-68). Les prêtres de SipeSipe, Tiquipaya et Colcapirhua, qui étaient certes les fonctionnaires en contact le plus intime avec la population des paroisses, n'utilisaient pas le terme cholo, lui préférant mestizo. Vers la fin du XVIIIe siècle, les prêtres de Passo l'abandonnèrent également. Comme nous venons de le voir, son emploi dans le rapport de Viedma était dicté par les impératifs fiscaux d'un fonctionnaire métropolitain doué de sens pratique.

L'indépendance de la Bolivie amena aussi des changements dans la désignation des populations non indigènes. Español fut remplacé par blanco, politiquement neutre. À peu près au même moment, les prêtres laissèrent tomber indio pour adopter indíjena. Ceux de Passo introduisirent blanco en 1836, au moment de la confédération entre le Pérou et la Bolivie, puis l'abandonnèrent (voir le tableau 7). Andrés Santa Cruz, leader responsable de la création de la confédération, était lui-même un mestizo, et son accession au pouvoir favorisa, pendant plusieurs années, le rejet des mentalités et des perceptions associées à plusieurs siècles de domination espagnole et au système de castes colonial. À SipeSipe, par ailleurs, les prêtres avaient adopté le terme blanco dès 1827 (APS). La chute de Santa Cruz et la restauration de l'oligarchie remit en usage les anciens termes raciaux.

Outre l'évolution de l'usage, deux facteurs sont source d'incertitude quant au sens des termes raciaux désignant les ruraux de la Valle Bajo: l'habiletê de certains pour négocier avec les prêtres le choix du terme qui devait définir le statut racial des membres de leur famille et la préférence personnelle des prêtres pour un terme ou l'autre, qui pouvait entraîner d'importants changements à court terme dans la distribution des baptêmes par catégorie. On trouve un exemple de négociation du statut racial dans le registre des baptêmes de Passo. En 1740, un nommé Benito de la Vega, identifié comme español, épousa une femme du nom d'Ascensia Condori, de la catégorie español également. Condori était un patronyme communément associé aux populations indigènes ou mestizos, mais non aux españoles. La même année, le couple fit baptiser un fils appelé Manuel de la Vega, qui fut inscrit comme español dans le registre des baptêmes. Ce cas attire l'attention sur un aspect de l'imprécision inhérente à l'attribution du statut racial. Biologi- 
quement, Ascensia Condori était indio ou mestizo, selon toute vraisemblance, mais il se peut qu'elle ait été issue d'une famille de haut rang, peut-être même qu'elle ait été la fille d'un Kuraka (chef d'une communauté indigène). Son mariage à un español permit de négocier le statut d'español pour elle aussi bien que pour son fils Manuel, qui était problablement d'ascendance mixte, mais était acceptê socialement comme español. De toute évidence, la situation sociale influençait le statut racial (APP).

Les gens ordinaires avaient moins d'influence, et les prêtres exerçaient donc dans leur cas un plus grand contrôle sur le choix du statut racial à assigner aux petits baptisés. Ils avaient chacun leurs préférences à cet égard, et en particulier il existe plusieurs exemples de prêtres de la même paroisse inscrivant plus d'enfants, les uns comme indios, les autres comme mestizos. Ces variations pouvaient aussi résulter de changements politiques. Le registre des baptêmes de Tiquipaya illustre clairement cette tendance dans les années 1830 , période où la rapidité du mestizaje se combine au souci du gouvernement d'êtablir avec précision la taille de la population indigène. Entre 1830 et 1835, la distribution des nouveau-nés enregistrés comme indios passa de 28 à 39,8 pour cent, et celle des mestizos de 56,2 à 62,2 pour cent. En 1836, après la création de la confédération Pérou-Bolivie et la promulgation par le gouvernement de règlements ordonnant d'enregistrer les naissances, les enterrements et les mariages des indigènes dans des registres distincts de ceux de la population non indigène, Mateo Caero, prêtre en poste à Tiquipaya, inscrivit un plus grand nombre de bébés indios. Cette année-là, il classa 46,2 pour cent des baptisés comme indios et 47,7 pour cent comme mestizos. L'année suivante, la distribution fut de 40,8 et de 51,7 pour cent respectivement. Puis, en 1838, Caero cessa d'enregistrer les baptêmes d'indios dans le même registre que les baptêmes des enfants non indigènes. Il avait évidemment réagi au changement de climat politique de 1836 et à l'accession au pouvoir d'Andrés Santa Cruz en définissant comme indios des enfants qui auraient sans doute passé pour mestizos l'année précédente. En 1837, revenant à la pratique antérieure à 1836, il avait classé plus de nouveau-nés comme mestizos.

Ces deux exemples de flottement dans l'attribution du statut racial laissent croire que le mestizaje peut jusqu'à un certain point résulter d'un tour de prestidigitation démographique. L'examen de l'endogamie et de l'exogamie dans les communautés de la Valle Bajo va nous fournir de nouveaux 
éléments pour montrer jusqu'où va l'imprécision. Les mariages entre personnes de même statut racial ou la tendance à attribuer le même statut racial aux deux partenaires d'un couple inciteraient à contester le postulat voulant que le mestizaje biologique ait été rapide et mettraient en lumière l'incertitude du statut racial consigné dans les registres.

\section{ENDOGAMIE ET EXOGAMIE}

La notion de mestizaje rapide suppose l'union fréquente de partenaires inscrits dans des catégories raciales différentes, par exemple d'españoles ou de mestizos avec des indios. L'étude des mariages donne une idée de l'ampleur du mestizaje, de la subjectivité du jugement des prêtres qui tenaient les registres et de l'habileté des couples à négocier le statut racial qui leur était attribué lors de la publication des bans et dans les registres, ainsi que des contradictions que recèlent ces derniers. Des taux d'endogamie élevés iraient de pair avec de faibles taux de mestizaje, ou manifesteraient à tout le moins la propension des prêtres à donner le même statut racial aux partenaires. Ils fourniraient aussi la preuve de la contradiction entre les registres des baptêmes et les registres des mariages (confirmant l'imprécision du processus d'attribution du statut racial), dans la mesure où les premiers laissent croire à un mestizaje rapide et où, contenant peu de mariages "interraciaux", c'est-à-dire d'unions entre indios et non indigènes, les seconds attesteraient plutôt la faiblesse des taux de mestizaje. À l'inverse, un nombre êlevé de mariages interraciaux soutiendrait l'hypothèse du fort mélange entre les groupes.

Le statut racial des couples figurait dans deux types de documents : les investigaciones matrimoniales et les registres des mariages. Les enquêtes sur les mariages permettaient de vérifier si les fiancés répondaient aux règles du droit canon et d'établir en particulier s'il existait des empêchements légaux à leur union. On publiait ou proclamait les bans dans la communauté pour solliciter de l'information sur le couple. Le registre des mariages contenait quant à lui divers renseignements comme le nom des parents des époux et, normalement, le statut racial de ces derniers et de leurs parents.

Je me suis servi d'échantillons tirés des registres des mariages de Passo et de SipeSipe. Respectivement constitués de 1404 et 1444 cas, ils comprennent toutes les unions enregistrées durant les brèves périodes retenues comme échantillon 
TABLEAU 8-Distribution des mariages endogames et exogames dans la paroisse de Passo, diverses années (en pourcentage)

\begin{tabular}{lccc}
\hline $\begin{array}{l}\text { Période } \\
\text { d'échan- } \\
\text { tillonnage }\end{array}$ & $\begin{array}{c}\text { Couples classés } \\
\text { dans la même } \\
\text { catégorie }\end{array}$ & $\begin{array}{c}\text { Couples classés } \\
\text { dans deux catégo- } \\
\text { ries différentes }\end{array}$ & $\begin{array}{c}\text { Catégorie } \\
\text { non } \\
\text { inscrite }\end{array}$ \\
\hline $1738-1742$ & 60,1 & 12,1 & 27,7 \\
$1750-1754$ & 86,2 & 12,6 & 1,3 \\
$1760-1764$ & 92,6 & 7,4 & - \\
$1770-1774$ & 76,4 & 23,6 & - \\
$1780-1784$ & 76,8 & 14,8 & 8,4 \\
$1790-1794$ & 69,7 & 24,6 & 1,6 \\
$1837-1841$ & 84,6 & 15,4 & - \\
$1876-1880$ & 75,3 & 24,2 & 0,5 \\
\hline
\end{tabular}

Source : Investigaciones matrimoniales de Passo et Registres des mariages de Passo, Archives paroissiales de Passo, Passo, Bolivie.

chronologique. Les échantillons de Passo datent de la fin des années 1730 aux années 1790, de 1837-1841 et de 1876-1980. Ceux de SipeSipe couvrent les périodes $1724-1728$ à 1764 1768, 1800-1804 et 1827-1831 (voir les tableaux 8 et 9).

Durant les premières décennies du XVIIIe siècle, le statut racial des époux n'est pas noté de façon systématique. Dans bien des cas, on ne fournit d'informations complètes que pour le mari. Notons que l'enregistrement des parents des nouveaunés souffre du même manque de cohérence. Dans l'échantillon 1738-1742 établi pour Passo d'après les investigaciones matri-

TABLEAU 9-Distribution des mariages endogames et exogames dans la paroisse de SipeSipe, diverses années (en pourcentage)

\begin{tabular}{lccr}
\hline $\begin{array}{l}\text { Période } \\
\text { d'échan- } \\
\text { tillonnage }\end{array}$ & $\begin{array}{c}\text { Couples classés } \\
\text { dans la même } \\
\text { catégorie }\end{array}$ & $\begin{array}{c}\text { Couples classés } \\
\text { dans deux catégo- } \\
\text { ries différentes }\end{array}$ & $\begin{array}{c}\text { Catégorie } \\
\text { non } \\
\text { inscrite }\end{array}$ \\
\hline $1724-1728$ & 8,7 & 1,0 & 90,3 \\
$1734-1738$ & 14,2 & 0,6 & 85,2 \\
$1744-1748$ & 81,9 & 15,9 & 2,1 \\
$1754-1758$ & 85,3 & 14,3 & 0,5 \\
$1764-1768$ & 78,9 & 20,6 & 0,6 \\
$1800-1804$ & 65,9 & 17,3 & 16,8 \\
$1827-1831$ & 83,9 & 15,7 & 0,4 \\
\hline
\end{tabular}

Source : Registres des mariages de SipeSipe, Archives paroissiales de SipeSipe, SipeSipe, Bolivie. 
moniales, 27,7 pour cent des inscriptions ne contiennent pas de renseignements complets sur le mari et sur la femme. Il en va de même pour la majorité des inscriptions des registres de SipeSipe selon les échantillons de 1724-1728 et de 1734-1738.

Durant la deuxième moitié du XVIIIe siècle, les prêtres des deux paroisses enregistrèrent le statut racial du mari et de la femme de façon assez systématique. Les deux échantillons présentent des taux élevés de mariages intraraciaux, mais on remarque aussi une baisse du nombre de couples identifiés par la même catégorie raciale. À Passo, sur quelque trente ans (entre l'échantillon de 1760-1764 et celui de 1790-1794), la proportion de couples inscrits dans la même catégorie passe en effet de 92,6 à 69,7 pour cent. Elle remonte à 84,6 pour cent en 1837-1841, puis redescend à 75,3 pour cent à la fin des années 1870. Dans les registres des mariages de SipeSipe, la fourchette se situe entre 85,3 pour cent d'endogamie (sommet atteint dans l'échantillon de $1754-1758$ ) et 78,9 pour cent (1764-1768). Clemente Boado y Quiróga ayant inscrit de très nombreux mariages sans préciser le statut racial des époux, en 1804, la fréquence des mariages intraraciaux tombe à 65,9 pour cent. Elle remontera à 83,9 pour cent dans l'échantillon de 1827-1831.

Le déclin de la proportion des unions entre conjoints de même race observé vers la fin du XVIIIe siècle peut être attribué à deux facteurs, soit une augmentation réelle du nombre de mariages de partenaires possédant ou déclarant un statut racial différent et - hypothèse plus plausible - la décision prise de leur propre chef par certains prêtres d'inscrire les époux dans des catégories différentes ou de reconnaître la validité du statut déclaré par eux. Ces choix personnels des prêtres sont détectables dans les registres des mariages, et ils sont susceptibles d'influencer davantage les résultats obtenus à partir des échantillons qui en sont tirés, étant donné leur taille inférieure, que ceux qui découlent des échantillons plus importants recueillis dans les registres des baptêmes. Par exemple, à SipeSipe, à la fin des années 1820, Manuel Olmos inscrit le même statut racial pour la plupart des couples, tandis que Miguel Frias fait l'inverse; de son côté, José Arze donne le même statut à tous les couples, sauf un.

Le taux apparemment élevé de mariages intraraciaux à l'intérieur des catégories artificielles créées dans les registres semble contredire l'hypothèse du mestizaje accéléré, qui fournirait l'explication la plus plausible de l'augmentation du nombre 
de jeunes enfants classés comme mestizos dans les registres des baptêmes et partant du déclin relatif du nombre d'indios. Or, la croissance de la population de Calle Bajo demeure modeste au long des XVIIIe et XIXe siècles et, si l'on en juge par les taux d'endogamie en apparence élevés auxquels nous avons affaire (mais il ne s'agit vraisemblablement que d'une apparence), la population de chacun des groupes subjectivement caractérisés par une catégorie raciale devrait s'être-accrue sensiblement au même rythme. La composition raciale relative de la population devrait être restée assez stable, à moins d'avoir été influencée par l'immigration et la migration. Pourtant, les documents montrent que le nombre de nouveau-nés classés comme mestizos s'est accru rapidement, surtout durant les décennies qui ont suivi l'indépendance de la Bolivie, malgré la persistance de taux d'endogamie apparente passablement élevés.

Pour vérifier le déclin relatif du nombre de mariages intraraciaux inscrits dans les registres, on peut faire appel à des recensements où figurent le statut racial assigné aux personnes et, dans certains cas, la structure familiale de la population. Nous examinerons ici deux recensements paroissiaux détaillés : un dénombrement de la population de SipeSipe et des communautés environnantes, effectué en 1798 (Gordillo Claure, 1987), et un recensement réalisé à Passo en 1823 (S. n.).

Ces recensements comprenaient plusieurs types d'information, sur la race, bien sûr, mais aussi sur l'état civil et sur l'unité familiale. Tous deux constatent un taux élevé d'endogamie. Celui de SipeSipe inscrit, en 1798, 193 couples sur 837 dans la même catégorie raciale, soit 83 pour cent de l'ensemble. De même, à Passo, le recensement de 1823 enregistre 448 couples de même catégorie raciale sur 540 , soit 82,9 pour cent du total.

Dans le Haut-Pérou-Bolivie au cours de la période coloniale, et durant encore quelque cinq décennies après l'indépendance du pays, le statut indio était à la fois racial et fiscal. Mis à part les registres des baptêmes, des sépultures et des mariages que devaient tenir toutes les paroisses, le gouvernement se renseignait sur la taille de la population indigène au moyen d'un autre type de documents, provenant de l'énumération des tributaires (Indiens soumis au tribut) de chaque division administrative. Les changements apportés au système du tribut, son déclin puis sa disparition entre le milieu et la fin du XIXe siècle contribuèrent à la diminution de la population d'indios, au 
moins sur le papier. La relation entre le mestizaje et l'évolution du système du tribut est l'objet de la section suivante.

\section{MESTIZAJE ET TRIBUT}

Le système colonial espagnol comportait un statut strictement fiscal reposant sur la création d'un nouveau statut racial : celui d' indio. En tant que libres vassaux de la couronne, les indigènes de l'Amérique hispanique êtaient soumis à l'obligation de payer le tribut. Dans la région andine, à cause de la migration interne et du déclin démographique dû notamment à la maladie, le système du tribut subit divers changements, en particulier la multiplication des catégories de tributaires, conçue pour tirer le maximum des indigènes qui avaient quitté leur communauté d'origine. L'évolution du système du tribut a attiré l'attention des chercheurs, mais certains d'entre eux s'intêressent surtout aux catégories fiscales dont se servaient les fonctionnaires coloniaux pour imposer un statut fiscal aux peuples indigènes et aux différences de fardeau correspondantes (Alcoser, s. d.). Certes, en montrant que certaines régions comptaient un grand nombre de forasteros, on met en évidence le phénomène des migrations internes. Toutefois, le recours aux catégories fiscales pour l'étude de la population indigène du Haut-Pérou-Bolivie braque la lorgnette sur les différences de statut fiscal tout en évacuant des similitudes - valeurs communes, persistance de l'ayni (êchange de travail) - ou des différences plus fondamentales, au chapitre de la langue, du costume et de l'organisation économique par exemple. Cette approche tend aussi à figer artificiellement les distinctions entre paysans de statut fiscal différent ${ }^{5}$.

La population tributaire des communautés de la Valle Bajo a évolué en trois siècles, comme le montre le tableau 10. Les années 1573 à 1786 virent diminuer le nombre d'originarios, tributaires soumis au fardeau le plus élevé, tandis que, tout au long de la décennie 1680 , le nombre de forasteros augmenta. À la fin du XVIIe siècle et au début du XVIIIe, divers fonction-

5 Pour une analyse dêtaillée du système du tribut dans le Haut-Pérou, voir Sanchez-Albornoz, 1978. Powers (1995) examine l'impact de la migration sur le système du tribut et sur le processus d'ethnogênèse (recréation des cultures ethniques). L'êtude de l'ethnogénèse atteste l'utilité, sur le plan conceptuel, de dépasser les classifications espagnoles pour éclairer la création d'identités culturelles ainsi que la recréation et la redéfinition des relations sociales au sein des collectivités. 
TABLEAU 10 - Nombre de tributaires par catégorie dans trois communautés indigènes de la Valle Bajo

\begin{tabular}{|c|c|c|c|c|c|c|c|c|c|}
\hline \multirow[b]{2}{*}{ Année } & \multicolumn{3}{|c|}{ Passo } & \multicolumn{3}{|c|}{ SipeSipe } & \multicolumn{3}{|c|}{ Tiquipaya } \\
\hline & $O$ & $F$ & $\mathrm{Y}$ & $\mathrm{O}$ & $F$ & $\mathrm{Y}$ & $\mathrm{O}$ & $F$ & $\mathrm{Y}$ \\
\hline 1573 & 684 & - & - & 819 & - & - & 502 & - & - \\
\hline 1683 & 113 & 232 & 82 & 65 & 222 & 99 & 66 & 257 & 71 \\
\hline 1786 & 38 & 192 & - & 68 & 333 & - & 90 & 312 & - \\
\hline 1792 & 108 & 128 & - & 156 & 264 & - & 143 & 290 & - \\
\hline 1804 & 99 & 103 & - & 133 & 235 & - & 142 & 330 & - \\
\hline 1878 & 127 & 121 & - & 216 & - & - & - & - & \\
\hline
\end{tabular}

Source : Sanchez-Albornoz, 1978 : 29, 163; Rodriguez Ostria, 1991; Gordillo Claure et del Rio, 1993 : tableau 6; Gordillo Claure, 1987.

$\mathrm{O}=$ originarios; $\mathrm{F}=$ forasteros; $\mathrm{Y}=$ yanaconas.

naires espagnols soumirent des propositions pour réformer le système du tribut. Après 1786, le gouvernement de la colonie puis le gouvernement de la Bolivie indépendante tentèrent d'en augmenter le rendement en créant un plus grand nombre d'originarios. Le premier cas attestê des effets de ces manipulations à Cochabamba date de 1793-1794. Cette année-là, 79 nouveaux originarios surgissent à Passo, 91 à SipeSipe et 67 à Tiquipaya. Une poignée de tributaires reçurent aussi des terres à Vacas, Mizque et Sicaya (Powers, 1995 : 185). De même, en 1844, le gouvernement confia à Francisco Sempertegui la mission de trouver des terres dans la Valle Bajo pour des originarios et des forasteros nouvellement désignés (Rodriguez Ostria, 1991).

Au milieu du XIXe siècle, on modifia de nouveau le système pour ressusciter le statut de reservado. Il s'agissait de personnes qui avaient des liens de parenté avec des originarios décédếs et se voyaient imposer les mêmes charges que ceux-ci en échange du droit aux terres communautaires. Des lois adoptées en 1834 et en 1842 firent apparaître de nouveaux reservados dans la Valle Bajo (il en figurait déjà sur les listes de tributaires de la fin de l'ère coloniale). On en comptait 60 à Passo, SipeSipe, Tiquipaya et Colcapirhua en 1845, 85 six ans plus tard, en 1851, 139 en 1858 et 132 en 1878 (Rodriguez Ostria, 1991 : 296, tableau 5).

Malgré ces détours destinés à remplir les listes de tributaires, l'analyse des registres du tribut donne l'impression d'un déclin général de la population indienne dans la Valle Bajo et dans toute la région de Cochabamba au cours du XIXe siècle. 
Entre 1852 et 1877, par exemple, la population indigène énumérée pour les fins du tribut passa de quelque 47287 à 29 155. Dans la province de Tapacari, où était située la Valle Bajo, la chute fut de 16847 à 10696 (Jackson et Maddox, 1993 : tableau 2). Mais cette apparence de déclin était une illusion engendrée par le système, plus qu'une preuve du recul démographique des indios. Exception faite du recensement national de 1900 et des quelques recensements effectués dans les paroisses, par exemple à SipeSipe en 1798 et à Passo en 1823, l'information dont on dispose sur la population indigène de la Bolivie provient essentiellement des recensements réalisés dans le cadre du système du tribut. La diminution du nombre de tributaires devait fatalement, à tout le moins dans les documents, s'accompagner de celle de la population indigène.

Le déclin du nombre de tributaires était le signe de l'échec du système dans la mesure où les intéressés se soustrayaient à l'obligation de payer. Ceux qui vivaient et travaillaient dans les haciendas échappaient à leur statut plus rapidement que ceux qui restaient sur les terres communautaires. Le taux global de déclin du nombre de tributaires entre 1838 et 1877 fut de 38 pour cent dans le département de Cochabamba, contre 57 pour cent pour les ouvriers des haciendas. Dans les provinces de Tapacari et de Cercado, qui comprenaient la Valle Bajo et les districts des montagnes voisines, le déclin atteignait respectivement 39 et 77 pour cent (Jackson et Maddox, 1993 : 270). Les listes de tributaires contenant moins de noms, la population d'indios sembla décliner.

Durant la deuxième moitié du XIXe siècle, les grands propriétaires fonciers de la Valle Bajo et des districts avoisinants durent faire face à de graves difficultés économiques causées par la réorientation des marchés régionaux, et nombreux furent ceux qui perdirent leurs terres. Pour réduire les coûts de production en éliminant à toutes fins utiles le coût du travail, ils recoururent à une forme de métayage voisine du servage. Les tenanciers échangeaient leur prestation de travail contre l'accès à un lot censé assurer leur subsistance, et certains payaient en outre un modeste loyer en argent ${ }^{6}$. Ils pouvaient ainsi échapper au tribut, et plus d'un propriétaire s'arrangea, par souci d'économie, pour faire retirer le nom de ses ouvriers

6 Pour une analyse globale de la transformation des marchés et des difficultés économiques auxquelles ont fait face les propriétaires d'hacienda de Cochabamba, voir Jackson, 1994. Sur l'économie du colonaje (mètayage), voir Jackson, 1988. 
des listes de tributaires, ce qui explique la chute rapide du nombre de tributaires vivant dans les haciendas de la Valle Bajo. La démarche des propriétaires leur évitait de payer le tribut imposé à leurs colonos, en vertu de la pratique instaurée durant la période coloniale. Par ailleurs, certains paysans de Cochabamba partirent travailler dans les mines de l'altiplano et de la région désertique de l'Atacama, sur la côte du Pacifique; cette migration s'est longtemps poursuivie au cours du XXe siècle ${ }^{7}$. Avec leurs économies, les mineurs étaient à même d'acheter, sur le marché, des biens - des vêtements par exemple - par lesquels ils se distinguaient des stéréotypes associés aux indios.

La population indigène diminua-t-elle durant la deuxième moitiê du XIXe siècle ? Les preuves disponibles tendraient à montrer que non. Nous avons vu qu'il y a plutôt lieu d'imputer l'illusion du déclin démographique des indios à la décroissance du nombre de tributaires sur les listes officielles, et que la réduction du nombre de personnes entrant dans la catégorie des indios soumis au tribut a été assimilée à celle du nombre d'indios. En outre, les personnes qui pouvaient se soustraire à l'inscription sur les listes de tributaires n'étaient vraisemblablement pas enregistrées comme indios dans les recensements, et ce fut probablement le cas pour le recensement national de 1900.

Le déclin du système du tribut dans la Valle Bajo coïncida avec la transformation structurelle des économies régionales, qui contribua à faire évoluer les modes d'occupation du sol, suscitant en particulier la multiplication des petits propriétaires exploitants, familièrement appelés piqueros. Cette évolution, sur laquelle nous reviendrons dans la section suivante, renforça la tendance à classer les paysans comme mestizos plutôt que comme indios. Les transformations de la société rurale de Cochabamba durant la deuxième moitié du XIXe siècle s'accompagnèrent d'une modification de la perception que se faisaient les élites de l'identité indio ou mestizo et de l'aspect physique correspondant. La croissance du nombre de piqueros appuya ce processus, puisque les indios étaient généralement associés aux terres communautaires et non à la propriété privée du sol, qui était d'inspiration européenne. L'abolition des communautés indigènes et le développement, dans la Valle Bajo, d'un marché foncier facilitant en particulier

7 Sur les modèles d'émigration de Cochabamba, voir Jackson et Gordillo Claure, 1993. 
l'acquisition de petits lopins de terre par les paysans engendrèrent l'apparition des piqueros, qui ne pouvaient, en vertu de son contenu social et culturel, porter le nom d' indios.

\section{OCCUPATION DU SOL ET MESTIZAJE}

Comme on l'a vu, l'évolution des modes d'occupation du sol amorcée autour des années 1840 transforma les perceptions des élites à l'égard de l'identité raciale de la population rurale de la Valle Bajo. Les haciendas constituées durant la période coloniale furent subdivisées en raison des difficultés économiques entraînées par la rêorganisation des marchés régionaux et de l'endettement croissant des propriétaires. Un marchẻ foncier dynamique se développa dans la Valle Bajo durant la deuxième moitié du XIXe siècle et un nombre considérable de petits lots fut transigé. Deux facteurs expliquent cette accélération des ventes de terrains. D'une part, l'importation de grandes quantités de blé de l'étranger en direction des marchés urbains de l' altiplano, traditionnellement approvisionnés par les producteurs de Cochabamba, avait fait tourner au désastre la situation économique de nombreux propriétaires d'hacienda. Les exploitations furent vendues et dans certains cas systématiquement loties et cédées par morceaux. D'autre part, l'abolition des communautés indigènes puis le fractionnement et la commercialisation des terres communautaires de la Valle Bajo firent suite à la passation, en 1874, de la loi d'ex-vinculación, première mesure mettant hors la loi la propriétê collective du sol caractéristique des indios. Après 1878 , les ventes et reventes d'anciennes terres communautaires se multiplièrent, et des centaines d'hectares de terrain changèrent de mains, souvent sous forme de petits lots (Rodriguez Ostria, 1991 : tableau 9).

Le nombre de piqueros s'accrut et les terres agricoles de la Valle Bajo furent rapidement subdivisées. Les tableaux 11 et 12 résument les données tirées de relevés de cadastre correspondant à certaines portions des anciennes terres communautaires de Passo et de SipeSipe. Llankenquiri (Passo) est un bon exemple de piqueria, district principalement occupé par de petits exploitants. Au total, 73,6 pour cent du sol appartenait à des propriétaires possédant moins de cinq hectares. La majorité des propriétaires fonciers de Payacollo (SipeSipe) étaient également des piqueros. Cent vingt-deux d'entre eux, possédant moins de cinq hectares, détenaient 71,6 pour cent des terrains du district. 
TABLEAU 11 - Répartition de la propriété du sol selon la taille des exploitations, district de Llankenquiri, vers 1924

\begin{tabular}{lrrrr}
\hline \multirow{2}{*}{$\begin{array}{l}\text { Superficie } \\
\text { des propriétés } \\
\text { (ha) }\end{array}$} & \multicolumn{2}{c}{ Propriêtaires } & \multicolumn{2}{c}{ Superficie possédée } \\
\cline { 2 - 3 } \cline { 5 - 6 } & $\mathrm{N}$ & $\%$ & $\mathrm{Ha}$ & $\%$ \\
\hline $0-0,99$ & 86 & 69,9 & $38,51,61$ & 26,9 \\
$1-4,99$ & 33 & 26,8 & $66,78,28$ & 46,7 \\
$5-9,99$ & 2 & 1,6 & $13,38,84$ & 9,4 \\
$10-49,99$ & 2 & 1,6 & $24,31,66$ & 17,0 \\
Total & 123 & 99,9 & $143,00,39$ & 100,0 \\
\hline
\end{tabular}

Source : Catastro de la Propiedad Rústica, Archivo de la Prefectura de Cochabamba, Province de Quillacollo, Canton de Passo.

a. Hectares, ares et centiares.

Les paysans de Cochabamba prenaient une part active aux marchés locaux et régionaux afin de gagner de l'argent pour acheter des terrains, vendant leurs produits agricoles ou leur travail. Les membres de l'élite locale constataient l'esprit d'entreprise des paysans, qui travaillaient et économisaient pour acquérir des lots, activités rarement associées aux indios. En 1895, un fonctionnaire qui venait de travailler à la préparation du nouveau cadastre de la vallée de Sacaba, contiguë à la Valle Bajo, écrit à ce propos :

L'indio, l'ouvrier agricole, veine inépuisable qui depuis l'époque coloniale a constamment nourri l'avarice et la cupidité du patron ou du propriétaire terrien, aspire aujourd'hui à l'indépendance; si l'occasion d'acquérir un lot se présente à lui [...] sans s'arrêter au

TABLEAU 12 - Répartition de la propriété du sol selon la taille des exploitations, district de Payacollo, vers 1924

\begin{tabular}{lrrrrr}
\hline \multirow{2}{*}{$\begin{array}{l}\text { Superficie } \\
\text { des propriétés } \\
\text { (ha) }\end{array}$} & \multicolumn{2}{c}{ Propriétaires } & & \multicolumn{2}{c}{ Superficie possédée } \\
\cline { 2 - 3 } \cline { 5 - 6 } & $\mathrm{N}$ & $\%$ & & $\mathrm{Ha}$ & $\%$ \\
\hline $0-0,99$ & 96 & 76,8 & $39,88,27$ & 32,7 \\
$1-4,99$ & 26 & 20,8 & $47,42,28$ & 38,9 \\
$5-9,99$ & 2 & 1,6 & $14,35,96$ & 11,8 \\
$10-49,99$ & 1 & 0,8 & $20,32,99$ & 16,7 \\
Total & 125 & 100,0 & $121,99,50$ & 100,0 \\
\hline
\end{tabular}

Source : Catastro de la Propiedad Rústica, Archivo de la Prefectura de Cochabamba, Province de Quillacollos, Canton de SipeSipe.

a. Hectares, ares et centiares. 
moindre calcul, il vend tout ce qu'il possède, et même les quelques couvertures de son lit, et paie le prix extravagant qui est l'enfant de sa noble aspiration à l'indépendance ${ }^{8}$.

Trois décennies plus tard, l'évolution de la sociétê rurale de Cochabamba inspire ce commentaire à Octavio Salamanca, important propriétaire terrien du canton de Passo et frère du futur président Daniel Salamanca :

La population des vallées comprend plus de mestizos que d'indios [...] au moins pour moitié, les propriêtaires du sol sont en fait des mestizos ou, ainsi que nous les appelons traditionnellement, des indios [...] et comme la terre ne suffit pas à les faire vivre, ils deviennent marchands. Tandis que les hommes cultivent la terre ou travaillent comme journaliers, les femmes élèvent des animaux, filent et tissent, fabriquent de la bière de maïs [...] vendent des produits agricoles et font du commerce dans les mines et dans les villes de l' altiplano ${ }^{9}$.

Des milliers de paysans de Cochabamba partirent aussi travailler dans les mines d'argent et d'étain de l'altiplano bolivien, les mines de nitrate du nord du Chili et les plantations de canne à sucre du nord de l'Argentine, et revinrent acheter des terrains à Cochabamba (Jackson et Gordillo Claure, 1993 : 742 et note 33).

Ce faisant, les paysans de Cochabamba ébranlèrent certaines conceptions élitistes du statut et de l'identité raciale; par leur esprit d'entreprise, en se procurant des terres, en devenant piqueros, ils appuyaient la mutation du système de castes en société de classes. C'est du moins la théorie que défendent Chance et Taylor à propos des villes de la fin de l'ère coloniale. Le vieux stéréotype de l'indio, lié à l'idée de caste, ne tenait

8 «El indio, colono de finca que desde la epoca del colonaje ha sido siempre la inagotable veta de la codicia y avaricia del patrón o propietario, siente hoy la aspiración de independarse; se le presenta la ocasión de adquirir un terrazgo [...]; no consulta ni para mientes en calculo alguno, vende sus semovientes, hasta las pocas cobijas de su cama y paga el precio caprichoso hijo de su noble aspiración de independencia." Rafael Tejada, "Informe de la Comision Rectificadora de Catastro de la Provincia de Chapare», cité par Rodriguez Ostria et Solares, $1990: 35$.

9 "Los valles estan mas poblados por mestizos que indios [...] los propietarios de tierra, al menos la mitad de ellos, son en realidad mestizos, o como tradicialmente los llamamos, indios [...] y como la tierra no les abastece para subsistir, se vuelvan comerciantes. Mientras los hombres trabajan la tierra o como jornaleros, las mujeres crian ganado, hilan y tejen, fabrican chicha $[\ldots]$ venden productos agricolas, y comercian en las minas $y$ ciudades del altiplano" (Salamanca, $1931: 185$ ). 
plus. Octavio Salamanca dit bien, dans son écrit polémique publié en 1931, que traditionnellement, jusqu'à ce qu'ils deviennent piqueros et petits propriétaires terriens, on connaissait les mestizos en tant qu' indios. Comme par magie, la notion de classe s'était tout doucement substituée au concept élitiste de caste pour caractériser la population rurale de la Valle Bajo. Des paysans qui auraient presque à coup sûr été appelés indios à la fin du XVIIIe siècle recevaient le nom de mestizos après avoir gagné les rangs des piqueros et quitté les communautés indigènes où la terre était propriété collective. L'évolution des définitions accompagnait les transformations de la société et de l'économie rurales de la Valle Bajo. Le processus se poursuivit durant les premières décennies du XXe siècle : après 1900, la part des indios dans l'ensemble de la population recensée continua de diminuer ${ }^{10}$.

Une analyse des données du recensement de 1900 pour certains districts de montagne du département de Cochabamba permet de mieux comprendre les liens entre identité raciale et mode d'occupation du sol. Malgré les efforts des autorités pour fractionner et lotir leurs terres sous l'empire de la loi d'exvinculación, les communautés indigènes de Challa (province de Tapacari) et de Quirquiavi (province de l'Arque) parvinrent à les conserver collectivement sous forme de propriété indivise (occupation pro-indiviso). Bien que l'occupation communautaire du sol ait été rendue illégale par la loi d'ex-vinculación, après la

10 Un échantillon des sépultures enregistrées dans la paroisse de Passo entre 1917 et 1921 atteste le déclin continu du nombre relatif de personnes identifiees comme indios. Sur 884 enterrements, 70 pour cent concernent des mestizos, 25 pour cent des indios et 4 pour cent des blancos (Registres des sépultures de la paroisse de Passo, APP). Les registres de Colcapirhua présentent la mème tendance à partir de la fin du XIXe siècle, bien que de façon moins concluante. Par exemple, sur 592 baptêmes enregistrés à Colcapirhua entre 1867 et $1871,469(79,2$ pour cent) étaient ceux d'enfants classés comme mestizos, 120 (20,3 pour cent) concernaient des enfants españoles et seulement $3(0,5$ pour cent) des indios. Mais ces chiffres pourraient ne pas refléter l'ensemble des baptêmes. Un nouveau registre commencé en 1874 porte une note précisant qu'il contient les baptèmes d'españoles et de mestizos, ce qui laisse croire qu'un registre distinct était tenu pour la population indigène, conformément au décret émis dans les années 1830 . Toutefois, il appert que ce dernier s'est perdu. Durant les anneees 1890 , certains prêtres ont même cessê d'enregistrer le statut (à la fois fiscal et racial) dans les registres des baptêmes de Colcapirhua. En 1895 et en 1896, par exemple, Eduardo Zambrana ne l'inscrit pas dans le cas d'un nouveau-né. Nicanor Rivera, en poste à Colcapirhua de 1896 jusque dans la première décennie du XXe siècle, reprend cette pratique (Registres des baptêmes de Colcapirhua, APCP). 
dêsastreuse Guerre du Pacifique (1879-1884), le gouvernement bolivien adopta un compromis qui permettait aux collectivités indigènes de jouir d'une forme modifiée de leur mode d'occupation ancestral en recourant à une autre formule déjà prévue dans la loi bolivienne. L'occupation pro-indiviso, par exemple, permettait aux familles de riches propriétaires terriens de préserver l'intégrité d'une hacienda et d'éviter de démembrer l'unité de production que constituait le domaine afin de liquider un héritage. En appliquant la fiction légale de l'indivision à certaines communautés indigènes boliviennes, le gouvernement faisait respecter la loi d'ex-vinculación sans alimenter la résistance des collectivités qui refusaient de subdiviser leurs terres comme l'exigeait la loi de 1874 (Jackson, 1994 : chapitre 2.)

Au recensement de 1900 , la population de Challa et celle de Quirquiavi furent massivement inscrites comme indigènes : à 83,8 pour cent dans le premier district, à 73,1 pour cent dans le second (Oficina Nacional de Inmigración, Estadistica, y Propaganda Geografica, 1902). La préservation par les paysans des deux districts du caractère collectif de leur mode d'occupation du sol allait directement dans le sens des stéréotypes que l'ère coloniale avait rattachés au statut indio. De nombreux piqueros vivant dans la Valle Bajo durant les premières décennies du XXe siècle avaient fait partie de communautés indigènes dissoutes dont les membres avaient reçu le droit à la propriêté d'un lot individuel au cours des années 1870 et 1880 , ou étaient les enfants de paysans qui avaient ainsi, par l'effet d'une loi, acquis un nouveau statut défini par la propriété individuelle d'une terre. En dépit de certaines diffërences culturelles extêrieures, dans le costume par exemple, les piqueros de la Valle Bajo et les paysans des collectivités de Challa et de Quirquiavi partageaient de nombreux traits culturels, telles la langue et des pratiques socio-économiques comme l'ayni. Le principal facteur qui les distinguait, faisant appeler les uns mestizos et les autres indios, était le mode d'occupation du sol.

\section{CONCLUSION}

À l'instar des sources où étaient enregistrés l'identité et le statut racial des populations au XVIIIe, au XIXe et au début du $X X e$ siècle, le savoir démographique a changé au fil du temps et s'est trouvé marqué par un haut degré d'imprécision et de subjectivité. Les prêtres et les agents de recensement avaient généralement une bonne idée des éléments qui permettaient de 
reconnaître un indio ou un mestizo, bien que la définition des termes ait évolué au cours du XIXe siècle. Dans un sens, les mestizos des districts ruraux de la Valle Bajo étaient des indigènes qui ne se comportaient plus comme des indios et dont la culture, notamment, avait changé en 400 ans. Jusqu'à la fin de la période coloniale, il n'y eut pas, dans les registres des quatre communautés étudiées ici, d'appellation normalisée pour désigner la population indigène, et les termes employés pour la population non indigène faisaient également l'objet d'un certain flottement.

Un certain nombre de facteurs ont influencé le choix des termes utilisés pour décrire le statut racial des nouveau-nés. Les prêtres avaient souvent une préfêrence pour certaines désignations. Les parents d'un nouveau-né à baptiser pouvaient aussi parfois négocier le statut racial de leur enfant. L'évolution politique et les impératifs fiscaux des gouvernements modifièrent également l'usage des termes raciaux, comme l'illustre l'emploi par l'intendant Francisco de Viedma du terme cholo pour classer des centaines de paysans à la fin des années 1780. Après l'indépendance de la Bolivie, en 1825, à mesure que les politiques des gouvernements changeaient, les prêtres remplacèrent par des mots politiquement neutres comme indijena et blanco des termes fortement connotés associés au régime colonial.

Or, les identités raciales fabriquées par les registres paroissiaux, les recensements paroissiaux et les registres du tribut masquent certaines réalités sociales et culturelles et font parfois commettre des erreurs d'interprétation aux chercheurs qui acceptent tel quel le statut racial inscrit dans les documents. La question qui se pose est celle de savoir s'il existait ou non de véritables distinctions sociales et culturelles entre les paysans de la Valle Bajo selon que les prêtres et les agents de recensement les classaient comme indios ou comme mestizos. Mais les deux termes étaient artificiels, reflétant avant tout les conceptions sociales de la période coloniale et les stéréotypes élitistes qui référaient à la notion de caste des coutumes propres aux communautés indigènes, leur façon de se vêtir par exemple, et surtout la propriété collective du sol, si éloignée de la tradition européenne de la propriété privée. Les transformations économiques du XIXe siècle dans la Valle Bajo menèrent à la modification de l'occupation du sol. La croissance du nombre de petits propriétaires jointe au déclin du système du tribut accéléra le processus de mestizaje, mais le démographe a 
intérêt à se rappeler qu'il y a dans celui-ci une part d'illusion, une sorte de tour de magie.

Les flottements et la subjectivité qui entachent les termes raciaux utilisés pour classer la population rurale de la Valle Bajo font en sorte que les distinctions artificielles inscrites dans les registres sont de peu d'utilité pour les chercheurs qui essaient d'établir l'évolution démographique de certains segments de population, telle la population indigène. Notre analyse de l'imprécision et des avatars des définitions de l'identité raciale met donc en question la validité des études démographiques menées sur d'autres parties de l'Amérique hispanique à partir de documents et de catégories de même nature.

\section{REFÉRENCES BIBLIOGRAPHIGUES}

ALCOSER, Phelipe de. S.d. "Padrón General de la Doctrina de San Pedro de SipeSipe», APS.

APC : Registres paroissiaux des baptêmes de Colcapirhua, tirés des Archives paroissiales de Colcapirhua, Colcapirhua, Bolivie.

APP : Registres paroissiaux des baptêmes de Passo, tirês des Archives paroissiales de Passo, Passo, Bolivie.

APS : Registres paroissiaux des baptêmes de SipeSipe, tirés des Archives paroissiales de SipeSipe, SipeSipe, Bolivie.

APT : Registres paroissiaux des baptêmes de Tiquipaya, tirés des Archives paroissiales de Tiquipaya, Tiquipaya, Bolivie.

DEMELAS, Marie-Danièle, 1981. "Darwinismo a la criolla: El darwinismo social en Bolivia, 1880-1910", Historia Boliviana, 1-2 : 55-82.

GOOTENBERG, Paul, 1991. "Population and Ethnicity in Early Republican Peru: Some Revisions", Latin American Research Review, 26, 3 : 109-157.

GORDILLO CLAURE, José, 1987. "Análisis de un padrón general de la Doctrina de San Pedro de SipeSipe (Cochabamba), 1798", EstudiosUMSS, 1, $1:$ 41-63.

GORDILLO CLAURE, José, et Mercedes DEL RIO, 1993. La Visita de Tiquipaya (1573): Analisis Etno-Demográfico de un Padrón Toledano. Cochabamba, UMSS-CERES-ODEC/FRE.

GRIESHABER, Erwin, 1985. «Fluctuaciones en la definicion del indio: Comparación de los censos de 1900 y 1950", Historia Boliviana, 5 : 45-65.

HALE, Charles, 1968. Mexican Liberalism in the Age of Mora, 18211853. New Haven et Londres, Yale University Press. 
JACKSON, Robert H., 1988. "Evolución y Persistencia del Colonaje en las Haciendas de Cochabamba", Siglo $X I X, 3,6$ : 145-162.

JACKSON, Robert H., 1994. Regional Markets and Agrarian Transformation in Bolivia: Cochabamba 1539-1960. Albuquerque, University of New Mexico Press.

JACKSON, Robert H., et Gregory MADDOX, 1993. "The Creation of Identity: Colonial Society in Bolivia and Tanzania", Comparative Studies in Society and History, 35, 2 : 263-284.

JACKSON, Robert H., et José GORDILLO CLAURE, 1993. "Formación, crisis y transformación de la estructura agraria de Cochabamba. El caso de la hacienda de Paucarpata y de la comunidad del Passo, 1538-1645 y 1872-1929", Revista de Indias, 53 : 723-760.

LANGER, Erick, 1988. "El liberalismo y la abolición de la comunidad indigena en el siglo XIX", Historia y Cultura, 14 : 59-95.

LANGER, Erick, et Robert H. JACKSON, 1990. "El liberalismo y el problema de la tierra en Bolivia (1825-1920)", Siglo XIX, 5, 10 : 9-32.

LARSON, Brooke, 1988. Colonialism and Agrarian Transformation in Bolivia: Cochabamba 1550-1900. Princeton, Princeton University Press.

OFICINA NACIONAL DE INMIGRACIÓN, ESTADÍSTICA, Y PROPAGANDA GEOGRÁFICA, 1902. Censo General de la Población de la Republica de Bolivia Según el Padronamiento de 1 de Septiembre de 1900. La Paz, Oficina nacional [...].

POWERS, Karen, 1995. Andean Journeys: Migration, Ethnogenesis, and the State in Colonial Quito. Albuquerque, University of New Mexico Press.

RODRÍGUEZ OSTRIA, Gustavo, 1991. "Entre reformas y contrareformas: las comunidades indigenas en el Valle Bajo Cochabambino (1825-1900)*, dans Heraclio BONILLA, éd. Los Andes en la Encrucijada Indios, Comunidades y Estado en el siglo XIX. Quito, Clasco : 277-334.

RODRIGUEZ OSTRIA, Gustavo, et Humberto SOLARES, 1990. Sociedad oligarquica, chicha y cultura popular. Cochabamba, Honorable Municipalidad de Cochabamba.

SALAMANCA, Octavio, 1931. El socialismo en Bolivia. Cochabamba, Bolivar.

SANCHEZ-ALBORNOZ, Nicolas, 1978. Indios y tributos en el Alto Perú. Lima, Instituto de Estudios Peruanos.

SKIDMORE, Thomas, 1974. Black Into White: Race and Nationality in Brazilian Thought. New York, Oxford University Press.

S. n., "Padrón de Almas [de] esta Doctrina del Paso 1823", Archives paroissiales de Passo, Passo, Bolivie.

VIEDMA, Francisco de, 1969. Descripción geográfica y estadística de la provincia de Santa Crúz de la Sierra. Cochabamba, Editorial Los Amigos del Libro. 


\section{RÉSUMÉ - ABSTRACT - RESUMEN}

JACKSON Robert H. - NAISSANCE ET MÉTAMORPHOSES DU SAVOIR DÉMOGRAPHIQUE : LE MESTIZAJE DES COMMUNAUTÉS INDIGENES DE LA VALLE BAJO DE COCHABAMBA, EN BOLIVIE

Au XVIe et au XVIIe siècle, les conquérants espagnols créèrent un éventail de dénominations raciales pour désigner et catégoriser, à des fins fiscales, les populations américaines d'ascendance mixte. Étant donné que ce système de castes reposait sur le postulat que la filiation sanguine était reconnaissable à la couleur de la peau et aux caractéristiques physiques, les renseignements démographiques recueillis sur ces bases furent constamment marqués par l'imprécision. De plus, leur sens se transforma au fil des décennies. Les prêtres et les agents de recensement interprétèrent les termes raciaux de façon subjective et les utilisèrent en fonction de leurs préfërences individuelles. Par exemple, il arriva que dans les registres d'une paroisse, après l'arrivée ou le départ d'un prêtre, la fréquence de l'une ou l'autre catégorie raciale présente, à court terme, des variations significatives. Les changements socio-économiques modifièrent aussi, à la longue, le sens des termes raciaux et la définition des statuts qui y étaient rattachés. La Valle Bajo de Cochabamba, en Bolivie, illustre ce processus : la multiplication des mestizos dans les trois communautés indigènes étudiées ici ne fut pas strictement affaire de mélange racial et doit être mise en rapport avec l'évolution des identités et des statuts assignés aux indios.

JACKSON Robert H. - THE CREATION AND CHANGING DEFINITION OF DEMOGRAPHIC KNOWLEDGE: THE CASE OF MESTIZAJE IN THE CORPORATE INDIGENOUS COMMUNITIES OF THE VALLE BAJO OF COCHABAMBA, BOLIVIA

In the sixteenth and seventeenth centuries Spanish officials and members of the Catholic church created a series of race-based caste terms designed to identify and categorize the peoples of mixed ancestry. The Spanish American caste system relied on the assumed ability of a census-taker or priest to define the blood lines of an individual based on skin color and physical characteristics. However, the demographic knowledge created from the caste system was imprecise, and changed over time. Individual priests and census-takers showed idiosyncratic preferences for the use of one or another caste term. There were, for example, significant short-term shifts in the registration of caste terms in parish registers related to the arrival and/or departure of priests. There were also long-term shifts in the meaning of terms and the definitions of status caused by socioeconomic changes. An example is the rapid rate of apparent mestizoization in the corporate indigenous communities of the Valle Bajo of Cochabamba, Bolivia. The rapid increase in the number of mestizos was related to changing definitions of the status and identity of indigenous peoples, and was not strictly caused by racial mixture. 
JACKSON Robert H. - EL NACIMIENTO Y LAS METAMORFOSIS DEL SABER DEMOGRÄFICO, O EL MESTIZAJE DE LAS COMUNIDADES INDÍGENAS DEL VALLE BAJO DE COCHABAMBA, EN BOLIVA

Durante los siglos XVI y XVII, los conquistadores españoles crearon toda una serie de denominaciones raciales para designar y categorizar a las poblaciones americanas de origenes mixtos, con fines fiscales. Puesto que este sistema de castas descansaba en el postulado que se reconocía la filiación sanguínea por el color de la piel y las características fisicas, los datos demográficos obtenidos eran cambiantes $e$ imprecisos. Además, su sentido fue transformándose con los años. Los curas y censistas interpretaban subjetivamente los términos raciales, que utilizaban en función de sus propias preferencias. Por ejemplo, llegó a suceder que en los registros de una parroquia, tras la llegada o la partida de un cura, las categorias raciales presentaran, a corto plazo, variaciones significativas. Con el tiempo, los sentidos de los términos raciales y la definición de los estatutos se vieron también modificados por los cambios socioeconómicos. El Valle Bajo de Cochabamba, en Bolivia, ilustra este proceso: la multiplicación de los mestizos en las tres comunidades indígenas estudiadas no se debió estrictamente a una mezcla racial, y debe relacionarse con la evolución de las identidades y los estatutos de los indios. 